\title{
INTRODUCTION
}

\section{Problèmes et orientations actuels en immunologie des Invertébrés}

\author{
par C. VAGO \\ Université des Sciences, F 34000 Montpellier \\ et Station de Recherches de Cytopathologie, I.N.R.A.-C.N.R.S., F 30380 Saint-Christol
}

Le Colloque que j'ai l'honneur d'introduire par cet exposé de synthèse est l'un des premiers à l'échelle internationale envisageant d'examiner d'une manière comparée les acquis et la potentialité d'un domaine qui, après une longue période d'évolution très lente, se trouve depuis peu à un niveau où les sollicitations d'ordre appliqué et les problèmes fondamentaux deviennent aigus.

Cette introduction pourrait être commencée en constatant qu'il était normal que dès les années 1920 certains auteurs tels que Métalnikoff, Paillot ou Glaser se soient interrogés sur l'existence de l'immunité chez divers Invertébrés, notamment chez les insectes. Cependant, on serait plus près de la réalité en disant qu'il était surprenant que cet aspect de la physiologie et de la pathologie des Invertébrés n'ait pas bénéficié d'une attention plus précoce et plus soutenue pendant longtemps. On doit, en effet, admettre que l'immunologie des Invertébrés montre un retard considérable dans son évolution par rapport à celle, explosive dès la fin de la guerre, de la plupart des domaines de la pathologie des Invertébrés.

Des sentiments semblables à ceux qui viennent d'être évoqués éprouvent sans aucun doute les immunologistes médicaux et vétérinaires lorsqu'ils essaient de comparer les connaissances sur les mécanismes de l'immunité chez les Invertébrés et chez les Vertébrés.

Il n'est pas envisagé d'analyser ici les raisons de ce décalage de certains côtés par trop évidentes si l'on pense à la puissance des motivations liées au rôle des réactions immunologiques pour la médecine et qui ont imposé des recherches intenses, organisées, soutenues et urgentes sur les aspects fondamentaux. Il semble cependant que d'une manière paradoxale c'est précisément le progrès considérable de l'immunologie des Vertébrés qui a été une raison d'hésitation dans l'immunologie des Invertébrés dans 
des phases de début. On doit penser ici à l'insistance avec laquelle l'immunité basée sur la réaction antigène-anticorps, déjà bien connue chez les Vertébrés, a été recherchée pendant des dizaines d'années chez divers insectes et d'autres Invertébrés avant de s'engager vers d'autres orientations.

Mais, lorsque l'on considère la situation actuelle, on est amené à constater que c'est précisément par cette obligation récente de chercher diverses voies pour reconnaître les mécanismes d'immunité chez les Invertébrés que ce secteur se trouve subitement au plein carrefour des tendances les plus modernes de l'immunologie d'aujourd'hui.

Ainsi, en immunologie humaine et animale, on remarque, actuellement, de plus en plus que le rôle des facteurs protecteurs non spécifiques et probablement non liés aux anticorps a été assez négligé pendant longtemps pour l'interprétation de la résistance vis-à-vis des agressions. Or, chez les Invertébrés, en particulier chez les insectes, l'effet Je telles substances est fréquemment noté. Certaines expériences évoquent le rôle possible d'actions enzymatiques, par exemple, celle du lyzosyme. En même temps, la recherche de substances porteuses d'une certaine immunité est devenue active à la suite de l'observation de protection de relativement courte durée, mais souvent remarquablement efficace contre les infections bactériennes ou virales chez divers insectes ou crustacés. Ces substances, tantôt supposées être protéiques, tantôt de petites molécules, ne semblent pas être assimilables aux anticorps. L'étude de leur nature, de leur genèse et de leur action constitue une orientation actuellement poursuivie dans plusieurs laboratoires.

En dépit de ces incertitudes sur la nature des substances recherchées, les données récentes permettent d'apercevoir une nette relation avec d'autres secteurs de l'immunologie du fait même de l'existence indéniable chez divers Invertébrés, et notamment chez les insectes d'une immunité acquise, même antivirale, en partie comparable dans son déroulement à ce que l'on connaît chez les Vertébrés mais non liée à des immunoglobulines.

Plusieurs orientations peuvent être évoquées en tant qu'éléments de liaisons importants à l'échelle de l'immunologie comparée. Ainsi, on doit souligner l'intérêt récent qui s'est manifesté en pathologie des Invertébrés vis-à-vis de l'interaction d'infections virales. L'un des aspects est constitué par la très grande tolérance des cellules vis-à-vis de la replication simultanée de plusieurs virus dans un même noyau ou même cytoplasme cellulaire, l'autre mettant en avant le problème de l'existence d'interferons dans les divers groupes d'Invertébrés. Or, en virologie générale, l'étude des interferons est de grande actualité et certains envisagent l'universalité de leur action dans le monde vivant y compris les végétaux. 11 paraît normal que par des efforts conjugués d'immunologistes de Vertébrés et d'Invertébrés soient recherchées activement des liaisons entre ces systèmes qui apparaissent peut-être comme des suites ou des voies parallèles dans la phylogenèse du monde animal.

La contribution possible de l'immunologie des Invertébrés prend aujourd'hui un sens très large à l'éclaircissement des problèmes fondamentaux de l'immunité. En effet, l'étude de la faculté de reconnaissance par un organisme du «soi» et du «non soi» vient de trouver un matériel de haute valeur chez divers Invertébrés depuis que cette 
faculté ou des variantes de celle-ci ont été reconnues non seulement chez les Arthropo. des mais aussi chez les Invertébrés les plus primitifs. Il n'est pas douteux que l'étude approfondie de la tolérance ou du rejet des greffes chez les Invertébrés pourra apporter des éléments utiles, d'ordre phylogénétique, à la connaissance du mécanisme d'un phénomène de grande actualité médicale.

En abordant les perspectives dans le domaine de l'immunité cellulaire, on ne doit d'abord pas oublier qu'un certain Metchnikoff a montré déjà, il y a cent ans, le service que peut rendre l'étude de l'hématologie des Arthropodes à l'immunologie générale.

Aujourd'hui, il semble important d'admettre qu'il n'est guère raisonnable de maintenir une séparation par trop nette entre l'immunité humorale et cellulaire comme cela se pratique souvent pour les Invertébrés. Il paraît au contraire de plus en plus indispensable d'étudier le rôle des hémocytes dans l'élaboration des principes sériques encore mal définis et de rechercher des types nouveaux d'immunocompétence.

$\mathrm{Ce}$ sont les réactions cellulaires dont l'évolution phylogénétique a été suivie de plus près dans les divers groupes d'Invertébrés. La reconnaissance de la phagocytose à un stade primitif de l'évolution des Invertébrés, la découverte chez les Arthropodes de types particuliers de réactions tels que l'attraction épicytaire et la prédominance de réactions hémocytaires multicellulaires partiellement apparentées aux granulomes de Vertébrés sont des éléments de grande valeur pour l'interprétation des moyens de défense celllulaire, même à un niveau élevé de l'évolution animale.

Certains aspects sont déjà retenus parallèlement chez les Vertébrés et les Invertébrés, comme l'acquisition par des cellules participant à la réaction de granulome, de la faculté de constituer un stimulus pour l'attraction d'autres cellules ou encore la participation de divers tissus, par voie de prolifération, aux réactions multicellulaires.

Enfin, l'obtention récente de la formation «in vitro » des granulomes dans des milieux de composition connue avec visualisation et enregistrement continus est d'une importance particulière pour l'immunologie comparée du fait qu'elle ouvre la voie à l'étude expérimentale précise des influences physiques, chimiques et pathologiques sur cette réaction immunitaire très générale.

Tous les aspects ici évoqués convergent vers une évaluation de nos connaissances pour l'immunologie comparée. Cependant, on ne doit pas retenir l'impression que c'est le seul profil des problèmes et des tâches d'actualité concernant l'immunologie des Invertébrés.

En particulier, il doit être souligné combien la pathologie et la parasitologie des Invertébrés souffrent du retard dans les connaissances fondamentales sur l'immunité chez les Arthropodes ou les Mollusques. Les répercussions sont nombreuses et importantes.

Certaines d'entre elles sont liées aux graves problèmes que posent en agriculture les insectes ou d'autres Invertébrés ravageurs des cultures et des forêts. Elles ont trait notamment à la recherche des facteurs intrinsèques qui président à la fluctuation des 
populations d'insectes nuisibles, en particulier, à leur régulation par les épizooties. Elles concernent aussi directement les applications pour lesquelles il serait primordial de connaître les réactions qu'opposent les populations d'insectes ravageurs aux traitements de lutte biologique par parasites ou par microorganismes.

En ce qui concerne les Invertébrés utiles, qu'il s'agisse de l'ostréiculture, conchyliculture, astaciculture, héliciculture, apiculture ou sériciculture, ou encore de la production d'insectes entomophages pour la lutte biologique, on s'interroge depuis longtemps sur les principes immunologiques susceptibles d'être exploités en vue de la protection contre les épizooties graves et économiquement très importantes.

Enfin, en épidémiologie humaine, animale ou végétale se pose constamment la question de la nature, en partie d'ordre immunitaire, de la tolérance sélective qui est à la base de la transmission de nombreux germes pathogènes, protozoaires ou helminthes parasites par des Arthropodes vecteurs et de la potentialité d'un vecteur d'acquérir les virus, rickettsies ou mycoplasmes transmis.

Les différents aspects évoqués montrent que l'immunologie des Invertébrés est aujourd'hui une discipline qui doit encore acquérir de nombreux éléments de base pour assurer son évolution. Pourtant les perspectives de cette dernière s'annoncent d'une manière de plus en plus précise.

D'un côté plusieurs sciences appliquées, telles que la pathologie, parasitologie, épidémiologie, zootechnie, phytiatrie réclament avec persistance l'éclaircissement de phénomènes d'ordre immunitaire qui faute de connaissances ne peuvent pas être pris en considération ou exploités.

D’un autre côté, le mécanisme des réactions immunitaires intéressant l'homme ne semble véritablement compréhensible qu'à la lumière des connaissances sur l'évolution phylogénétique de l'immunité aussi bien humorale que cellulaire.

Pour ces deux raisons, il paraît impensable que l'immunologie des Invertébrés puisse se développer si elle reste une discipline aussi isolée qu'elle l'est depuis près d'un siècle. Le présent Colloque constitue l'une des tentatives, encore rares, envisageant l'examen de certains problèmes et acquisitions relatifs à l'immunité des Invertébrés en commun avec les spécialistes de l'immunologie générale et médicale, de divers secteurs de la parasitologie, de la virologie, et de l'oncologie. Il est, en effet, indéniable qu'aujourd'hui cette discipline peut s'insérer valablement et sur plusieurs plans évoqués plus haut dans les tendances modernes de l'immunologie générale, notamment en apportant des éléments sur l'évolution phylogénétique des principes immunitaires et sur des types d'immunité particulière probablement jamais recherchée chez l'homme et les Vertébrés, en participant à l'étude des circuits d'organismes à faces multiples tels que ceux des germes et des parasites transmis par des vecteurs et en offrant toute sa potentialité de recherche sur « modèles» représentée par les avantages expérimentaux bien connus de beaucoup de « matériel invertébré ». 